\title{
Capsulorrexe em duplo anel: estudo clínico patológico
}

\section{Double ring capsulorhexis: clinic opathologic study}

Eduardo Ferrari Marback', Marcelo Siqueira de Freitas², Fernanda Tanajura Spínola ${ }^{3}$, Luciano Espinheira Fonseca Junior ${ }^{4}$

\section{RESUMO}

Apresentamos caso de capsulorrexe em duplo anel em paciente feminina de 81 anos. O espécime cirúrgico foi submetido a estudo anatomopatológico que evidenciou os achados típicos da capsulorrexe em duplo anel. Comentamos a possível relação com a exfoliação capsular verdadeira. Salientamos que é possível realizar a cirurgia com bom resultado mesmo que o duplo anel não seja completo - como no caso apresentado -, porém desde que os folhetos capsulares estejam fundidos de forma contínua.

Descritores: Extração da catarata; Catarata/patologia; Capsulorrexe; Cápsula do cristalino/cirurgia; Cápsula do cristalino/ patologia; Relatos de casos

\section{ABSTRACT}

We present a case of double ring capsulorhexis in a 81 year-old female patient. Surgical specimen was submitted to histopathologic study resulting in typical double ring capsulorhexis findings. We discuss the questioned relation to true capsular exfoliation. We call attention to the fact that it is possible to obtain a good surgical result even when the double ring is not complete-as the case presented -, since both capsular flaps are fused in a continuous form.

Keywords: Cataract extraction; Cataract/pathology; Capsulorhexis; Lens capsule/surgery; Lens capsule/pathology; Case reports

\footnotetext{
'Doutor em Oftalmologia, professor adjunto de Oftalmologia da Universidade Federal da Bahia (UFBA) e Cirurgião do Instituto de Olhos Freitas - Salvador (BA), Brasil;

${ }^{2}$ Mestre em Oftalmologia, diretor médico do Instituto de Olhos Freitas - Salvador (BA), Brasil;

${ }^{3}$ Médica, aluna do $3^{\circ}$ ano do Curso de Especialização em Oftalmologia da Universidade Federal da Bahia (UFBA) - Salvador (BA), Brasil;

${ }^{4}$ Doutor em Patologia Humana, professor adjunto de Anatomia Patológica da Universidade Federal da Bahia (UFBA) - Salvador (BA), Brasil.

*Trabalho realizado na Universidade Federal da Bahia e Instituto de Olhos Freitas
}

O autor declara não haver conflitos de interesse

Recebido para publicação em: 5/10/2011 - Aceito para publicação em: 21/12/2011 


\section{INTRODUÇÃO}

C om a popularização da capsulorrexe como capsulotomia de preferência para a cirurgia da catarata, começaram a surgir relatos do sinal de capsulorrexe em duplo anel (CDA). ${ }^{(1-3)}$ Alguns autores questionam se tais pacientes são portadores de estágio subclínico de exfoliação verdadeira da cápsula e se a CDA seria um possível sinal de fragilidade capsular com possíveis complicações cirúrgicas relacionadas. ${ }^{(2-4)}$

Apresentamos um caso de CDA, incluído seus aspectos clínicos e estudo anatomopatológico.

\section{Relato do caso}

Paciente feminina, 81 anos, dona de casa, queixava-se de piora progressiva da visão em ambos os olhos (AO). Ao exame apresentava melhor acuidade visual corrigida igual a visão de vultos em olho direito (OD) e 20/100 em olho esquerdo (OE). À biomicroscopia revelou catarata nuclear ++/IV em AO. Mapeamento de retina evidenciando degeneração macular relacionada à idade do tipo não exsudativa em $\mathrm{AO}$, mais avançada em OD. Foi submetida à facoemulsificação com implante de lente intraocular em OD sem intercorrências, com melhora da acuidade visual para 20/400. Na cirurgia do OE foi notada ocorrência de duplo anel durante a confecção da capsulorrexe, iniciando-se no meridiano das 10horas (Figura 1). Ao perceber a presença do segundo anel, foi tomado o cuidado para manter a integridade e continuidade dos dois "flaps", que voltaram a unir-se no meridiano das 03 horas. A porção excisada da cápsula anterior foi fixada em fomaldeído a $10 \%$ e enviada para estudo anatomopatológico. O restante da cirurgia transcorreu sem complicações, com lente intraocular bem posicionada dentro do saco capsular ao final. A acuidade visual pós-operatória melhorou para 20/50.

O estudo anatomopatológico do fragmento de cápsula anterior revelou a presença de delaminação superficial em extremidades, presença de áreas de rarefação do epitélio capsular e presença de vacúolos intraepiteliais (Figura 2), achados análogos àqueles encontrados na exfoliação verdadeira da cápsula do cristalino, caracterizando do ponto de vista anatomopatológico a situação conhecida como CDA.

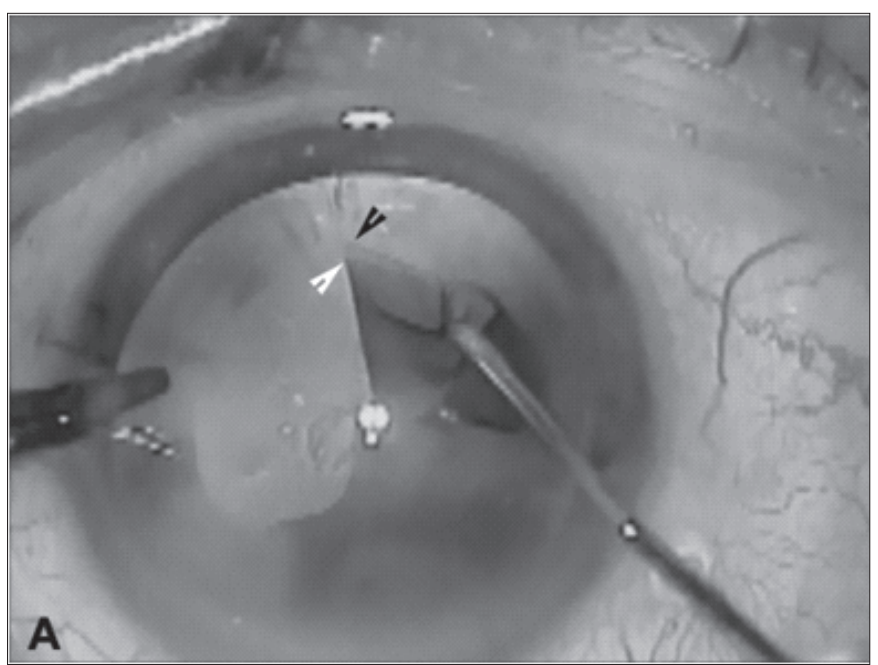

\section{DisCUSSÃO}

A incidência de CDA não é bem conhecida. Existe grande divergência na literatura com números que variam entre 5 até $40 \%$ das cirurgias de catarata. ${ }^{(2,3)}$ Acreditamos que esta variação tão grande talvez aconteça pelo desconhecimento de alguns cirurgiões sobre a existência de CDA, uma vez que está não é facilmente identificada em pequenos aumentos, especialmente se não foi utilizado corante vital, como o azul tripan, para realçar a cápsula anterior.

Alguns autores consideram a CDA como estágio subclínico da exfoliação verdadeira da cápsula. ${ }^{(1-3)}$ Tal afirmação é fundamentada no aspecto anatomopatológico indistinto entre as duas condições. ${ }^{(1-5)}$ Apesar dessa semelhança, Yamamoto et al., avaliando um grupo de 13 olhos com exfoliação capsular verdadeira e 11 com CDA, argumentaram que a CDA seria uma iatrogênia causada pela ação mecânica do manuseio sobre estrutura lamelar da cápsula do cristalino. ${ }^{(5)}$ Como reforço a esse pensamento, os autores comentam a apresentação de casos de delaminação capsular induzida pela injeção de substância viscoelástica na câmara anterior. ${ }^{(6)}$ Além disso, ao examinarem uma série de cápsulas de pacientes sem CDA os autores encontraram vacúolos no epitélio capsular e consideraram a presença de fendas na estrutura capsular e áreas de rarefação epitelial como artefatos de fixação. ${ }^{(5)}$

Embora não exista um fator de risco ambiental conhecido que predisponha a ocorrência da CDA, como a exposição a altas temperaturas predispõe a exfoliação verdadeira, os pacientes relatados com CDA habitualmente são idosos com mais de 80 anos. $^{(1-6)}$ Nesta faixa etária a exfoliação verdadeira chega a acometer $3,6 \%$ dos indivíduos, sendo relatada ainda uma maior ocorrência de pseudoexfoliação capsular e fragilidade zonular nos mesmos pacientes. ${ }^{(5)}$

A despeito da dúvida sobre a natureza da CDA como precursora ou não da exfoliação verdadeira, o estudo anatomopatológico deste e de outros casos de CDA não deixa dúvidas quanto à presença de fragilização capsular. ${ }^{(1-3,5)}$ Esta fragilização, por redução da espessura da cápsula anterior remanescente após a ocorrência da delaminação, justifica a argumentação para a execução de uma capsulorrexe contínua nos dois anéis. ${ }^{(1-3)} \mathrm{Em}$ nosso caso, o duplo anel não foi completo, e

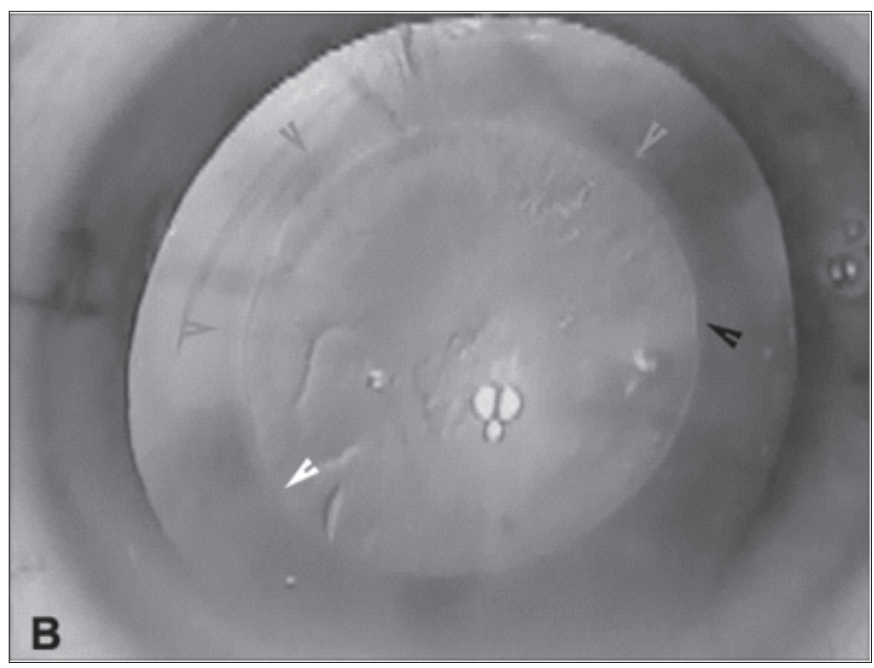

Figura 1: Aspecto transoperatório - A) Evidência da capsulorrexe em duplo anel durante sua execução; Folheto interno (seta branca); folheto externo (seta negra); B) capsulorrexe completa; notar o início do duplo anel (seta branca) e fim do duplo anel com fusão dos folhetos (seta negra) e limites do duplo anel (setas cinza) 

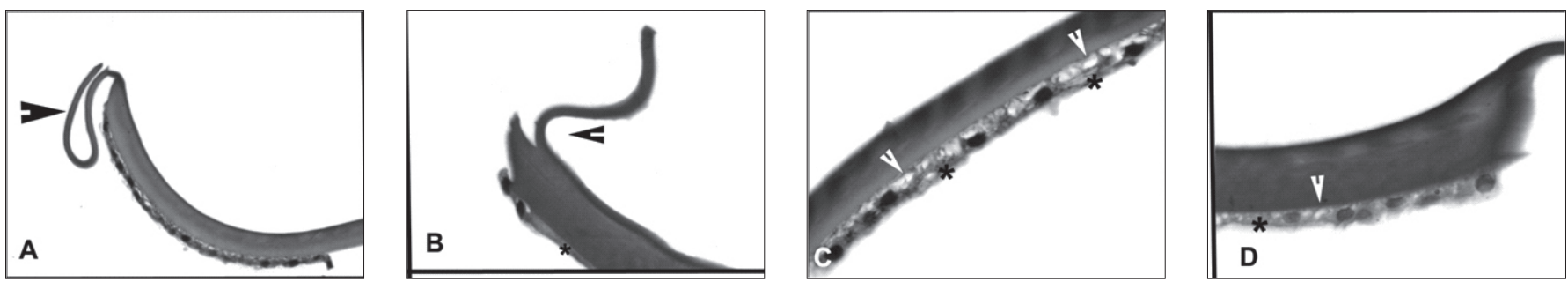

Figura 2: A) Delaminação da porção superficial da cápsula (seta) (PAS 200X); B) Detalhe da delaminação da cápsula (seta) e rarefação do epitélio (asterisco)(PAS 400X); C e D) Presença de vacúolos claros no citoplasma do epitélio (setas) e rarefação de células epiteliais (asteriscos) (PAS 400X)

começou a acontecer delaminação visível no meridiano das 10 horas, continuando-se até o meridiano das 3 horas. A partir daí,o "flap" de cápsula anterior assumiu contorno único, permitindo a continuidade da cirurgia e sua finalização com lente intraocular implantada no saco capsular. Diante disto, chamamos a atenção não para a necessidade de executar um duplo anel completo, mas sim para a necessidade de continuidade do corte sem lesar a lamela superficial antes que a delaminação termine, permitindo assim que não exista um ponto de fraqueza isolado que predisponha ao escape periférico da capsulorrexe.

\section{REFERÊNCIAS}

1. Braude LS, Edward DP. Partial splitting of the anterior lens capsule giving a 'double-ring' sign. ArchOphthalmol. 1995;113(6):705-8.

2. Ataka S, Kohno T, Kurita K, Wada S, Takahashi Y, Shiraki K. Histopathological study of the anterior lens capsule with a double-ring sign. Graefes Arch ClinExpOphthalmol. 2004;242(3):245-9.

3. Wollensak G, Wollensak J. Double contour of the lens capsule edges after continuous curvilinear capsulorhexis. GraefesArch ClinExpOphthalmol. 1997;235(4):204-7.
4. Marback EF, Rolemberg BM, Silva Filho HG, Araújo RCA, Freitas MS. Exfoliação capsular verdadeira:relato de dois casos. ArqBras Oftalmol. 2008;71(5):719-21.

5. Yamamoto Y, Nakakuki T, Nishino K, Yagyu K, Kishi S, Ueno H, Fukushima A.Histological and clinical study of eyes with true exfoliation and a double-ring sign on the anterior lens capsule. Can J Ophthalmol. 2010;44(6):657-62.

6. Kulkarni AR, Al-Ibrahim J, Haider S, Elsherbiny S, Scott R. Phacoemulsification in true exfoliation of the lens capsule: a case series. Eye (Lond). 2007;21(6):835-7.

\section{Autor correspondente:}

Eduardo Marback

Rua Eduardo José dos Santos, no 147 - Sala 808

CEP 41940-455 - Salvador (BA), Brasil

Fone /Fax: (71) 3235-9368

E-mail: eduardomarback@uol.com.br 